\title{
Synthesis of (Poly-methyl Methacrylate-lead Oxide) Nanocomposites and Studying their A.C Electrical Properties for Piezoelectric Applications
}

\author{
Dalal Hassan, Ahmed Hashim \\ University of Babylon, College of Education for Pure Sciences, Department of Physics, Iraq
}

\begin{tabular}{l} 
Article Info \\
\hline Article history: \\
Received March 14, 2018 \\
Revised May 21, 2018 \\
Accepted Oct 08, 2018 \\
\hline
\end{tabular}

Keywords:

Dielectric properties

Lead oxide

Nanocomposite

Poly-methyl methacrylate

\begin{abstract}
Piezoelectric materials have been prepared from (poly-methyl methacrylatelead oxide) nanocomposites for electronic applications. The lead oxide nanoparticles were added to poly-methyl methacrylate by different concentrations are $(4,8$, and 12$) \mathrm{wt} \%$. The structural and dielectric properties of nanocomposites were studied. The results showed that the dielectric constant and dielectric loss of nanocomposites decrease with increase in frequency of applied electric field. The A.C electrical conductivity increases with increase in frequency. The dielectric constant, dielectric loss and A.C electrical conductivity of poly-methyl methacrylate increase with increase in lead oxide nanoparticles concentrations. The results of pressure sensor showed that the electrical resistance of $\left(\mathrm{PMMA}^{\left.-\mathrm{PbO}_{2}\right)}\right.$ nanocomposites decreases with increase in pressure.
\end{abstract}

Copyright $(2018$ Institute of Advanced Engineering and Science. All rights reserved.

Corresponding Author:

Ahmed Hashim,

Department of Physics,

College of Education for Pure Sciences,

University of Babylon, Iraq

Email: ahmed_taay@yahoo.com

\section{INTRODUCTION}

The advances in the fundamental materials science lead to the development of new functional materials or new combination of existing materials, which exhibit superior electrical and optical properties. Researchers are enforced to focus on composites which consist of polymer as host matrix and semiconductor particles as filler due to the practical interest towards the better electrical, optical and mechanical properties of individuals and its dielectric, ferroelectric and piezoelectric properties are widely used in various applications. Recently, composite membranes are becoming prominent candidates in an intensive development of transducers, energy harvesting devices, underwater hydrophones, embedded capacitors, highpressure sensors, biomedical imaging and non-destructive testing applications. The most important materials used for wireless communication devices are microwave dielectric materials with appropriate permittivity, low dielectric loss and a suitable temperature coefficient of resonant frequency. It is for this reason that composite dielectrics and its electrical and optical properties have been so intensively investigated. Moreover, there is easiness in the preparation of polymer/semiconductor composite with diverse properties and hence tailor the properties as per requirements [1]. The rapid development of electronic products and power equipment, functional materials with excellent thermal and electrical properties are highly desirable. Polymeric materials are attractive due to their light weight, flexibility, ease of processing, and most importantly low cost. Interesting electrical applications need the electrical conductivity of a polymeric matrix to be improved by the addition of conductive particles. The charge carriers density will increase leading to formation of a conductive or semi-conductive composite Particularly filled composites are commonly used in several applications. This is because introducing fillers, in particular nanoparticles, enhances the thermal and electrical properties of the polymer composites. The addition of nano-fillers conforms to the chain length of the polymer and then reduces Maxwell-Wagner-Sillar type interfacial polarization originated from the 
differences in dielectric permittivity of the polymer and filler. There is an interest in dielectric materials because of their wide range of applications in electronic and electrical industry. High dielectric constant, dielectrics, are suitable materials for cable insulation, charge-storage capacitors, electrocaloric cooling, and artificial muscles [2]. Most of polymer materials have very high electrical resistance. In electronic industries, polymer materials are being widely used to produce numerous parts such as circuit boards, IC chip trays, encapsulants for electronic device, housings of personal computers, frames of monitors, keyboards, mouses and so on. Many of these applications take the advantages of polymer materials as good insulators, but some applications require polymer materials to be electrically conductive [3]. Polymeric materials have attracted the scientific and technological researchers, because of their wide applications. This is mainly due to the lightweight, good mechanical strength, optical properties and makes them to be multifunctional materials. In recent years, studies of electrical and optical properties of the polymer have attracted much attention in view of their application in electronic and optical devices [4]. Pressure sensors have a great significance for industrial equipment and they are widely used for the control and monitoring of thousands of applications, such as biomedical, environment, space, and automobiles. Many works have been conducted on pressure measurement using various techniques and three major types of pressure sensors have been investigated using capacitive, piezoelectric, and piezoresistive measurements. For example, capacitive pressure sensors use a diaphragm and a pressure cavity to create a variable capacitance for detecting strain by applying pressure. Common diaphragm materials are metal, ceramic, and silicon. Capacitive pressure sensors have been reported to be suitable for low-pressure measurements because of their sensitive diaphragms, but the linearity of their capacitance is poor due to the parasitic issue during the measurement. For piezoelectric pressure sensors, the piezoelectric effect in certain materials, such as quartz, has been used to measure the strain. This technology is commonly employed to measure highly dynamic pressure phenomena but it is insensitive to static responses. Furthermore, piezoresistive pressure sensors have been proposed to detect the strain by applying pressure to change the resistance of test patterns. Materials typically used for piezoresistive pressure sensors are silicon, polysilicon thin films, bonded metal foils, sputtered thin films, and inkjet printing films. Generally, piezoresistive pressure sensors are the most commonly employed technology in the pressure sensor market owing to their advantages of high sensitivity and low cost [5].

\section{EXPERIMENTAL PART}

The nanocomposites of polymethyl methacrylate-lead oxide were prepared by dissolving $2 \mathrm{gm}$ of poly-methyl methacrylate in $20 \mathrm{ml}$ of chloroform by using magnetic stirrer to mix the polymers for 1 hour. The lead oxide $(\mathrm{PbO} 2)$ nanoparticles added to polymethyl methacrylate with different concentrations are (4, 8 and 12) wt.\%. The casting method was used to prepare the samples of nanocomposites. The samples prepared with thickness range (220-240) $\mu \mathrm{m}$. The dielectric properties of (PMMA-PbO2) nanocomosites samples were examined in frequency range from $100 \mathrm{~Hz}$ to $5 \times 106 \mathrm{~Hz}$ by using LCR meter type (HIOKI 3532-50 LCR HI TESTER). The pressure sensor application of nanocomposites was investigated by measuring the resistance between two electrodes on the top and bottom of the sample for different pressures range (80-200) bar. The dielectric constant ( $\dot{\varepsilon})$ of nanocomposites is given by using the following (1) [6]:

$$
\dot{\varepsilon}=\frac{\mathrm{C}_{\mathrm{p}}}{\mathrm{C}_{s}}
$$

Where; $\mathrm{C}_{\mathrm{p}}$ is parallel capacitance and $\mathrm{C}_{\mathrm{o}}$ is vacuum capacitor

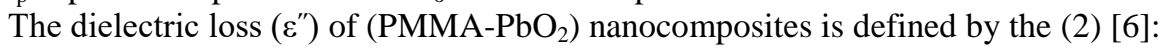

$$
\varepsilon^{\prime \prime}=\varepsilon^{\prime} \mathrm{D}
$$

Where, D: is dispersion factor of nanocomposites

The A.C electrical conductivity of $\left(\mathrm{PMMA}^{\left.-\mathrm{PbO}_{2}\right)}\right.$ nanocomposites is determined by the following (3) [7]:

$$
\sigma_{\mathrm{A} . \mathrm{C}}=\mathrm{W} \varepsilon " \varepsilon
$$

Where, w is the angular frequency. 


\section{RESULT AND DISCUSSION}

Figures 1 to 3 shows the variation of dielectric constant, dielectric loss and A.C electrical conductivity with frequency respectively. The dielectric properties of composites at a low frequency can be explained by interfacial polarization or Maxwell- Wagner-Sillars effect. The polarization spends much more time than other dielectric processes because of the inertia of formed dipoles. Hence, the interfacial polarization occurs at a low frequency, dielectric constant and dielectric loss decrease rapidly when frequency increases. At higher frequencies, the response from PMMA matrix becomes dominant, the dielectric constant is comparatively independent of frequency and is generally lower than that at a low frequency. The AC conductivity is frequency dependent and enhanced with an increase of the frequency. This can be accounted for by assuming that there may be charge carriers, which can be transported by hopping through the defect sites along the polymer chain [8].

As shown in Figures 1 to 3, the dielectric constant, dielectric losses, and AC electrical conductivity of the PMMA increase with the concentration of lead oxide nanoparticles. This result can be attributed to an increase in the conductivity as a result of the increase in the charge carrier density in the polymer matrix and the space charge polarization contribution [9] as shown in Figure 4.

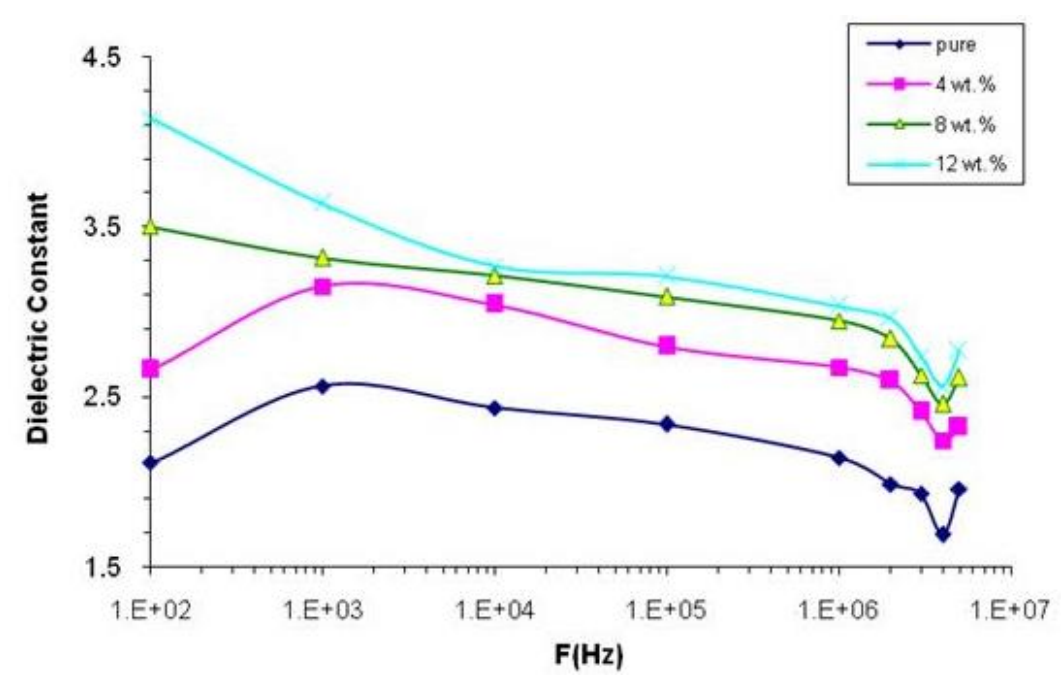

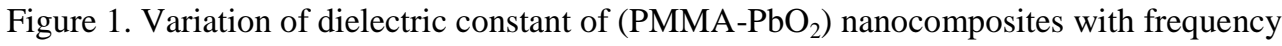

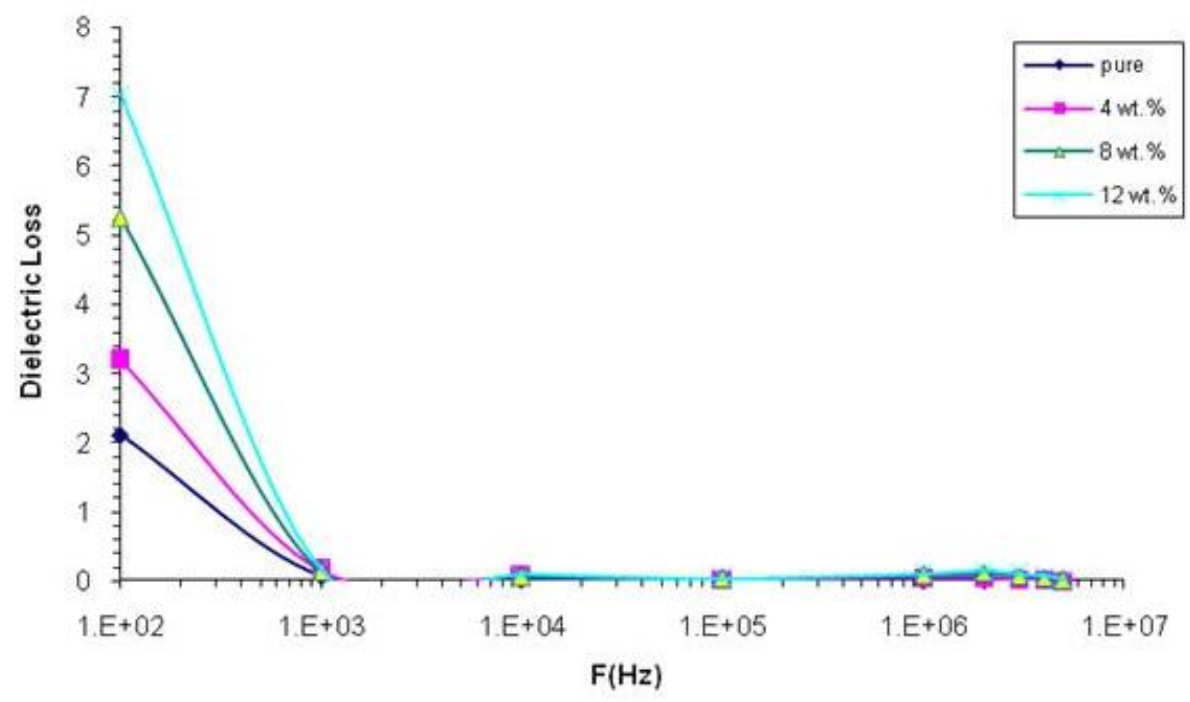

Figure 2. Variation of dielectric loss of ( $\left.\mathrm{PMMA}-\mathrm{PbO}_{2}\right)$ nanocomposites with frequency 


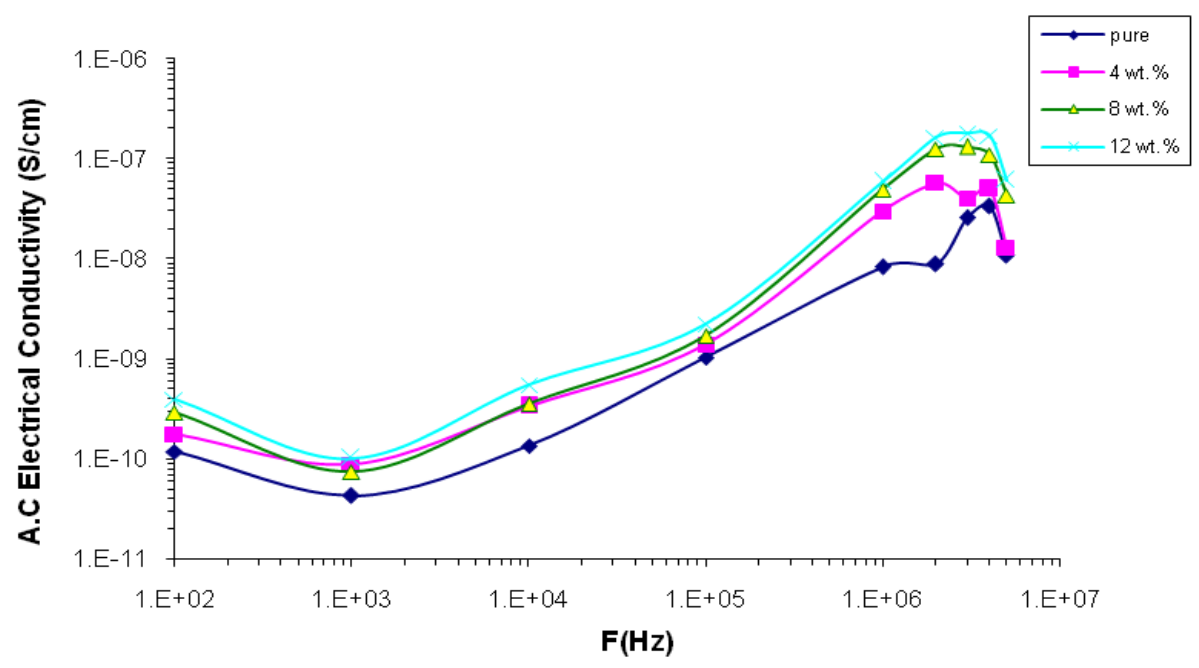

Figure 3. Variation of A.C electrical conductivity for ( $\left.\mathrm{PMMA}-\mathrm{PbO}_{2}\right)$ nanocomposites with frequency

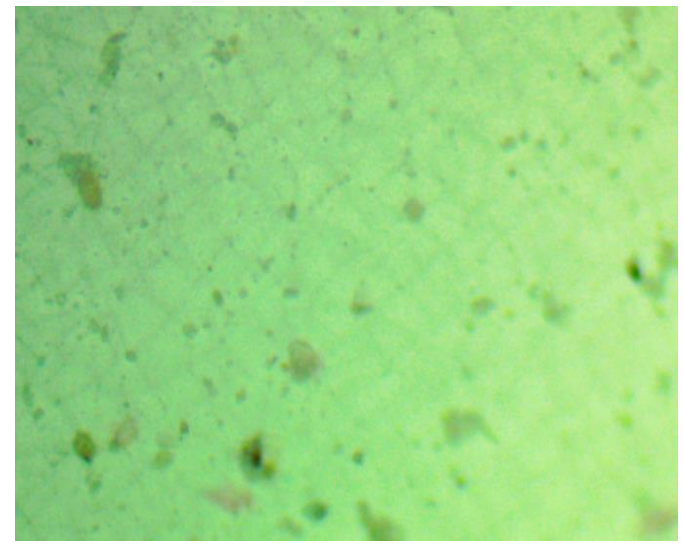

(a)

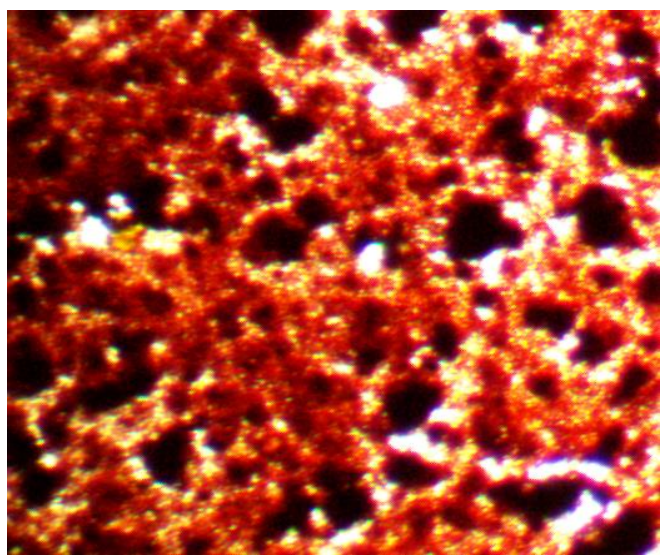

(c)

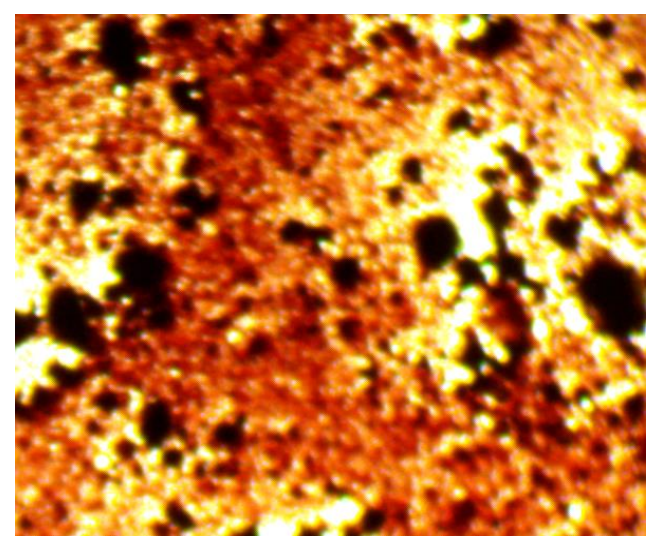

(b)

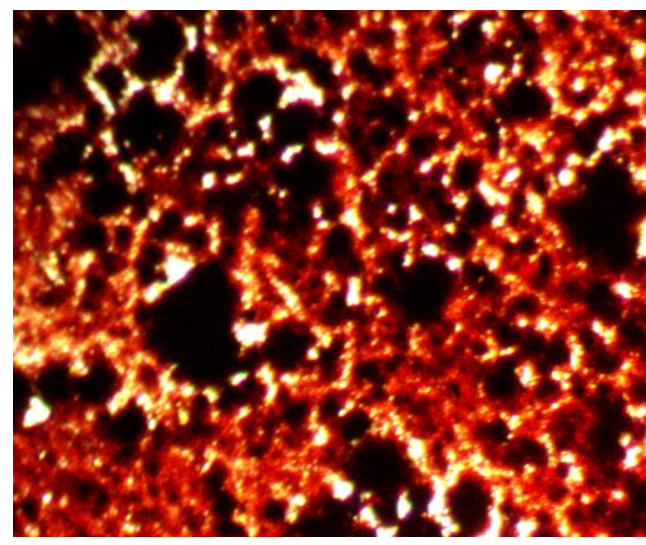

(d)

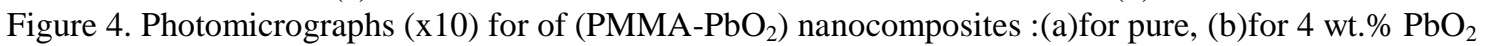
nanoparticles, (c)for 8 wt. $\% \mathrm{PbO}_{2}$ nanoparticles, (d)for $12 \mathrm{wt} \% \mathrm{PbO}_{2}$ nanoparticles

Figure 5 shows the variation of electrical resistance for $\left(\mathrm{PMMA}-\mathrm{PbO}_{2}\right)$ nanocomposites with pressure. As shown in Figure, the electrical resistance of nanocomposites decreases as the pressure increases. The samples have a crystalline region that has an internal dipole moment. These dipole moments are randomly oriented without any mechanical or electrical poling process, and the net dipole moment is zero in 
this condition. When stress is applied, it will change the local dipole distributions and induce an electric field. The induced electric field accumulates the charges at both the top and bottom of the sample [10].

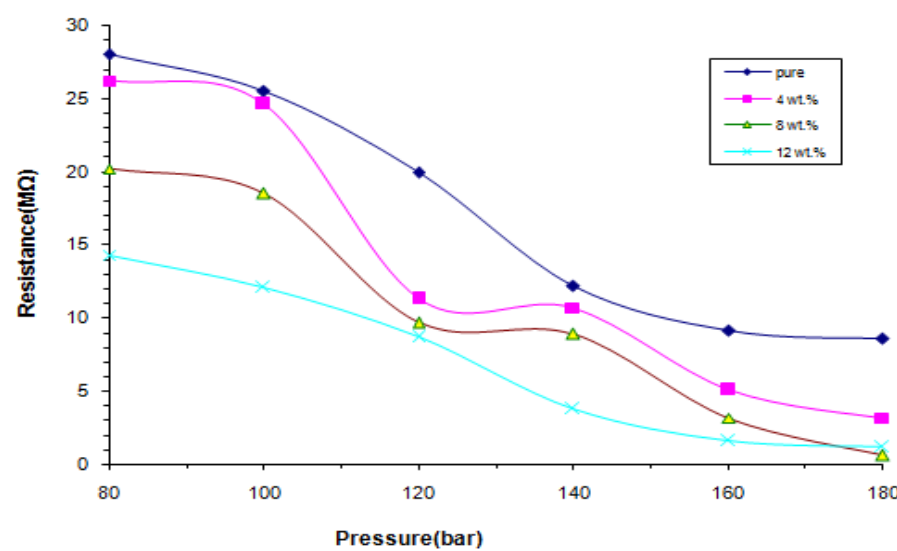

Figure 5. Variation of electrical resistance for $\left(\mathrm{PMMA}-\mathrm{PbO}_{2}\right)$ nanocomposites with pressure

\section{CONCLUSION}

a. The dielectric constant, dielectric loss and A.C electrical conductivity of poly-methyl methacrylate increase with increasing of the lead oxide nanoparticles concentration.

b. The dielectric constant and dielectric loss of (poly-methyl methacrylate-lead oxide) nanocomposites decrease with increasing of the frequency while the A.C electrical conductivity increases with increasing of the frequency.

c. The application of ( $\left.\mathrm{PMMA}-\mathrm{PbO}_{2}\right)$ nanocomposites as piezoelectric materials shows the nanocomposites

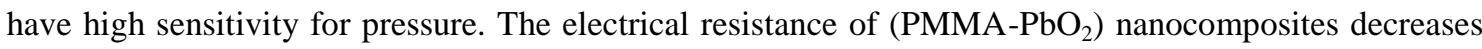
with increase in the pressure which are may useful for piezoelectric or pressure sensors with low cost and low weight.

\section{REFERENCES}

[1] Jobin Job Mathen, Ginson P. Joseph, J. Madhavan, Improving the optical and dielectric properties of PVA matrix with nano-ZnSe: Synthesis and Characterization, IJEDR, Vol. 4, Issue 3, (2016).

[2] L. A. Abdelwahab, Amr El-Hag Ali, R. A. Zaghlool, N. A. Mohsen, Dielectric properties, Impedance Analysis, and Electrical Conductivity of Ag Doped Radiation Grafted Polypropylene, Egypt. J. Rad. Sci. Applic., Vol. 30, No.1, (2017).

[3] Reda, S.M. and Sheikha M. Al-Ghannam, enhanced dielectric properties of polyaniline/silver/carbon nanotubes nanocomposite, American Journal of Nanotechnology, Vol.4, No.1, (2013).

[4] Alaa J. Kadham, Dalal Hassan, Najlaa Mohammad, Ahmed Hashim, Fabrication of (Polymer Blend-magnesium Oxide) Nanoparticle and Studying their Optical Properties for Optoelectronic Applications, Bulletin of Electrical Engineering and Informatics, Vol. 7, No. 1, (2018).

[5] Jer-Chyi Wang, Rajat Subhra Karmakar, Yu-Jen Lu, Chiung-Yin Huang and Kuo-Chen Wei, Characterization of Piezoresistive PEDOT:PSS Pressure Sensors with Inter-Digitated and Cross-Point Electrode Structures, Sensors, Vol.15, (2015): 818-831.

[6] Ahmed Hashim, Ibrahim R. Agool and Kadhim J. Kadhim, Novel of (Polymer Blend-Fe3O4) Magnetic Nanocomposites: Preparation and Characterization For Thermal Energy Storage and Release, Gamma Ray Shielding, Antibacterial Activity and Humidity Sensors Applications, Journal of Materials Science: Materials in Electronics, DOI 10.1007/s10854-018-9095-z, (2018).

[7] Jagadish Naik, R. F. Bhajantri, Sunil G. Rathod, Synthesis and characterization of multifunctional ZnBr2/PVA polymer dielectrics, journal of advanced dielectrics, Vol. 6, No. 4, (2016).

[8] L. A. Abdelwahab, Amr El-Hag Ali, R. A. Zaghlool, N. A. Mohsen, Dielectric properties, Impedance Analysis, and Electrical Conductivity of Ag Doped Radiation Grafted Polypropylene, Egypt. J. Rad. Sci. Applic., Vol. 30, No.1 (2017): 95-107.

[9] A. Hashim, A. Hadi, synthesis and characterization of novel piezoelectric and energy storage nanocomposites: biodegradable materials-magnesium oxide nanoparticles, Ukrainian Journal of Physics, Vol. 62, No.12, (2017).

[10] Ahmed Hashim and Qassim Hadi, Novel of (Niobium Carbide/Polymer Blend) Nanocomposites: Fabrication and Characterization for Pressure Sensor, Sensor Letters, Vol.15, (2017). 